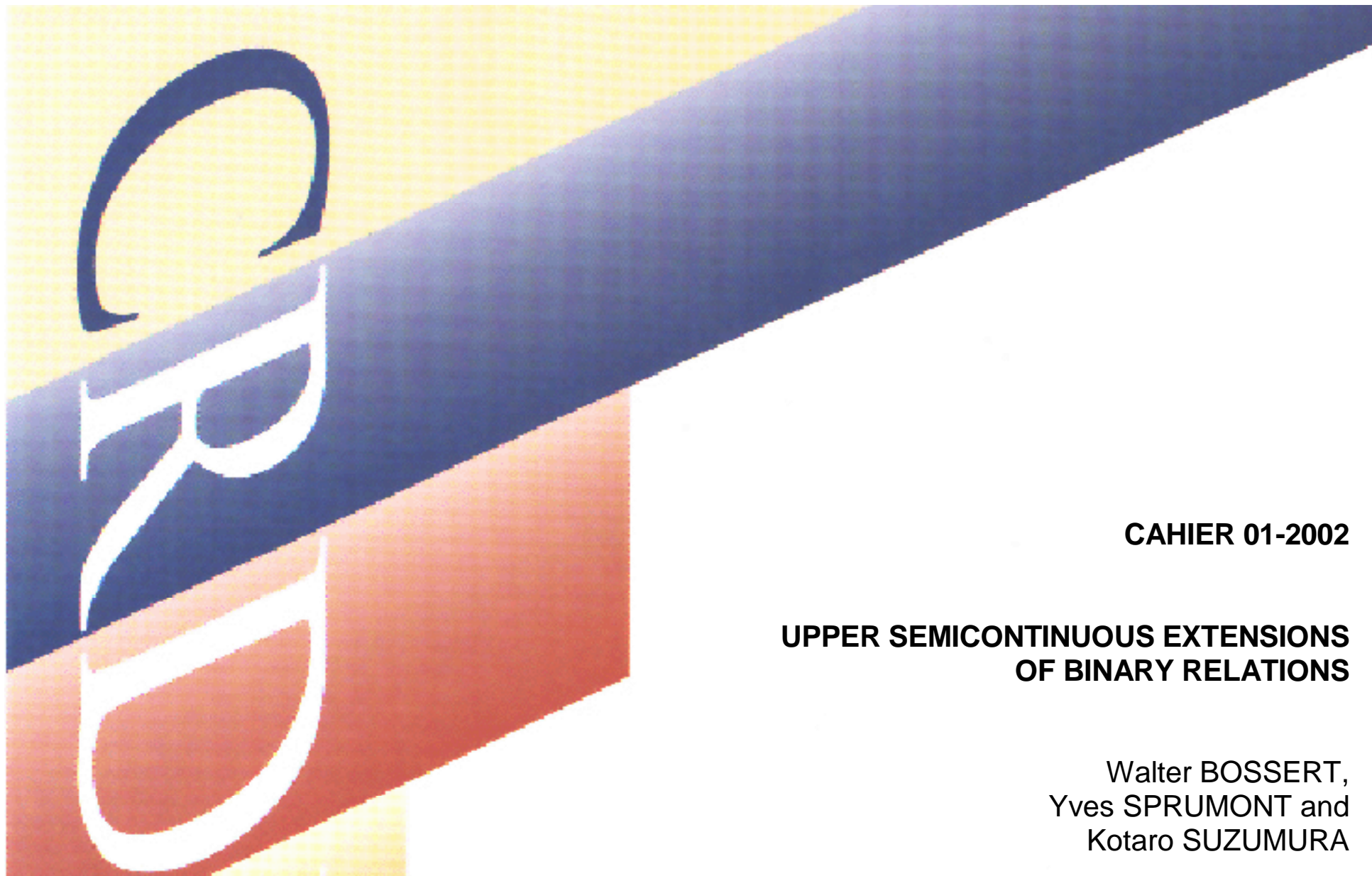

Centre de recherche

et développement en économique

C.P. 6128 , succursale Centre-ville

Montréal QC H3C 3J7

Téléphone : (514) 343-6557

Télécopieur : (514) 343-5831

crde@crde.umontreal.ca

http://www. crde umontreal.ca/

Université $\mathrm{th}$
de Montréal 
CAHIER 01-2002

\title{
UPPER SEMICONTINUOUS EXTENSIONS OF BINARY RELATIONS
}

\author{
Walter BOSSERT ${ }^{1}$, Yves SPRUMONT ${ }^{1}$ and Kotaro SUZUMURA ${ }^{2}$
}

1 Centre de recherche et développement en économique (C.R.D.E.) and Département de sciences économiques, Université de Montréal

2 Institute of Economic Research, Hitotsubashi University

January 2002

Financial support through grants from the Social Sciences and Humanities Research Council of Canada, the Fonds pour la Formation de chercheurs et l'aide à la recherche of Québec, and a Grant-in-Aid for Scientific Research for Priority Areas (B) Number 603 from the Ministry of Education, Science and Culture of Japan is gratefully acknowledged. 


\section{RÉSUMÉ}

Suzumura montre qu'une relation binaire peut être étendue à un ordre faible si et seulement si elle est cohérente. La cohérence n'est cependant ni nécessaire ni suffisante pour qu'une relation binaire semi-continue supérieurement puisse être étendue à un ordre faible semi-continu supérieurement. Jaffray montre qu'une relation binaire asymétrique (ou réflexive) transitive et semi-continue supérieurement peut être étendue à un ordre strict (ou faible) semi-continu supérieurement. Nous proposons des conditions qui assurent qu'une relation cohérente, plutôt que transitive, peut être étendue en respectant la semi-continuité supérieure. Si la relation est asymétrique, il suffit qu'elle soit cohérente et semi-continue supérieurement. Pour les relations qui ne sont pas asymétriques, nous prouvons un théorème d'extension qui utilise une condition de cohérence supplémentaire et un autre qui utilise une condition de continuité supplémentaire.

Mots clés : extensions, semi-continuité supérieure, cohérence

\section{ABSTRACT}

Suzumura shows that a binary relation has a weak order extension if and only if it is consistent. However, consistency is demonstrably not sufficient to extend an upper semi-continuous binary relation to an upper semicontinuous weak order. Jaffray proves that any asymmetric (or reflexive), transitive and upper semicontinuous binary relation has an upper semicontinuous strict (or weak) order extension. We provide sufficient conditions for the existence of upper semicontinuous extensions of consistent rather than transitive relations. For asymmetric relations, consistency and upper semicontinuity suffice. For more general relations, we prove one theorem using a further consistency property and another with an additional continuity requirement.

Key words : extensions, upper semicontinuity, consistency 


\section{Introduction}

It often makes sense to model preferences as binary relations that are not complete. Among many plausible reasons for allowing for incompleteness, Aumann (1962) lists the extreme complexity and the highly hypothetical nature of certain choice problems.

One way of assessing whether a preference relation is rational is to check whether it can be extended to a transitive and complete relation. Szpilrajn (1930) proves that every asymmetric and transitive relation has a strict order extension, and Hansson (1968) shows that every reflexive and transitive relation possesses a weak order extension. For detailed proofs of those results, see Fishburn (1970, pp. 16-18 and 1973, pp. 198-199, resp.); generalizations are discussed in Dushnik and Miller (1941), Donaldson and Weymark (1998), Duggan (1999) and Bossert (1999).

It turns out, however, that some binary relations that are not fully transitive may be extended as well. Suzumura (1976 and 1983, pp. 16-17) shows that a necessary and sufficient condition for the existence of a weak order extension is that the binary relation to be extended be consistent. Consistency requires that no preference cycle can contain a strict preference.

If a preference relation is defined on a topological space, it is of interest to extend it in a way that preserves its continuity properties. In particular, the existence of best elements in compact sets is guaranteed if a transitive and complete extension is upper semicontinuous. Jaffray (1975) proves that any upper semicontinuous and transitive relation possesses an upper semicontinuous strict order extension. Moreover, he shows that upper semicontinuous, reflexive and transitive relations have upper semicontinuous weak order extensions.

This paper investigates to what extent binary relations that are not fully transitive possess upper semicontinuous extensions. Since consistency is necessary for the existence of transitive and complete extensions, we focus on consistent relations. After stating Jaffray's result on extending asymmetric, transitive and upper semicontinuous relations, we prove three extension theorems. First, we weaken transitivity to consistency and show that the conclusion of Jaffray's theorem - the existence of an upper semicontinuous strict order extension - remains true. Because asymmetry is a very restrictive assumption in many economic environments (especially in conjunction with a continuity requirement), we examine the extent to which the asymmetry assumption can be dispensed with in the presence of consistency. However, for relations that are not necessarily asymmetric, consistency and upper semicontinuity together are not sufficient for the existence of an 
upper semicontinuous weak order extenson, and we illustrate this observation with an example.

We provide two sets of sufficient conditions for the existence of an upper semicontinuous weak order extension. The first is obtained by adding a property we call IP\&PIcomparability to consistency and upper semicontinuity. This axiom requires that whenever there is a preference chain of length two involving one strict preference and one indifference, the first and last elements in the chain must be comparable.

Whereas IP\&PI-comparability is a consistency-type condition, an alternative sufficiency result utilizes a further continuity axiom in addition to consistency and upper semicontinuity. This IP\&PI-continuity property requires that, for any alternative $x$, the set of alternatives $y$ such that there exists a $z$ which is indifferent to (resp. strictly preferred by) $x$ and strictly preferred to (resp. indifferent) to $y$ is open. As is the case for IP\&PI-comparability, IP\&PI-continuity can be added to consistency and upper semicontinuity in order to obtain a set of sufficient conditions for the existence of an upper semicontinuous weak order extension.

\section{Notation and basic concepts}

The set of positive (nonnegative) integers is denoted by $\mathbb{N}\left(\mathbb{N}_{0}\right)$. Let $X$ be a nonempty universal set of alternatives, and let $R \subseteq X \times X$ be a binary relation on $X$. The asymmetric factor $P(R)$ of $R$ is given by

$$
(x, y) \in P(R) \Leftrightarrow(x, y) \in R \text { and }(y, x) \notin R
$$

for all $x, y \in X$. The symmetric factor $I(R)$ of $R$ is defined by letting, for all $x, y \in X$,

$$
(x, y) \in I(R) \Leftrightarrow(x, y) \in R \text { and }(y, x) \in R .
$$

The noncomparable factor $N(R)$ of $R$ is defined by

$$
(x, y) \in N(R) \Leftrightarrow(x, y) \notin R \text { and }(y, x) \notin R
$$

for all $x, y \in X$.

The following are some standard properties commonly imposed on binary relations.

Reflexivity: For all $x \in X,(x, x) \in R$.

Asymmetry: For all $x, y \in X,(x, y) \in R \Rightarrow(y, x) \notin R$.

Transitivity: For all $x, y, z \in X,[(x, y) \in R$ and $(y, z) \in R] \Rightarrow(x, z) \in R$. 
Completeness: For all $x, y \in X$ such that $x \neq y,(x, y) \in R$ or $(y, x) \in R$.

Let $D=\{(x, x) \mid x \in X\}$ denote the diagonal relation on $X$. The transitive closure of $R \subseteq X \times X$ is denoted by $\bar{R}$, that is, for all $x, y \in X,(x, y) \in \bar{R}$ if there exist $K \in \mathbb{N}$ and $x_{0}, \ldots, x_{K} \in X$ such that $x=x_{0},\left(x_{k-1}, x_{k}\right) \in R$ for all $k \in\{1, \ldots, K\}$ and $x_{K}=y$. Clearly, $\bar{R}$ is transitive and, because the case $K=1$ is included, it follows that $R \subseteq \bar{R}$.

For a relation $R \subseteq X \times X, R^{*} \subseteq X \times X$ is an extension of $R$ if $R \subseteq R^{*}$ and $P(R) \subseteq P\left(R^{*}\right)$. If, in addition, $R^{*}$ is reflexive, transitive and complete, $R^{*}$ is called a weak order extension of $R$. Analogously, for an asymmetric relation $R \subseteq X \times X$, a relation $R^{*} \subseteq X \times X$ is a strict order extension of $R$ if $R^{*}$ is an asymmetric, transitive and complete relation such that $R \subseteq R^{*}$.

For simplicity of notation, we use the term topological space to refer to a set $X$ on which a topology is defined rather than to the pair consisting of $X$ and an associated topology. If $X$ is a topological space, we can define the property of upper semicontinuity for a relation $R \subseteq X \times X$.

Upper semicontinuity: For all $x \in X$, the set $\{y \in X \mid(x, y) \in P(R)\}$ is open in $X$.

\section{Extending asymmetric relations}

We begin with a restatement of the part of Jaffray's (1975) extension theorem that is relevant for our analysis. A proof can be found in Jaffray (1975, pp. 398-401).

Theorem 1 Let $X$ be a topological space, and let $R \subseteq X \times X$ be a binary relation on $X$. If $R$ is asymmetric, transitive and upper semicontinuous, then $R$ has an upper semicontinuous strict order extension $R^{*} \subseteq X \times X$.

In Jaffray's formulation, an additional order denseness property is imposed to obtain a corresponding property of $R^{*}$. Because we focus on the extension part of his theorem, this assumption is not required for our purposes.

Our first generalization of Jaffray's result retains asymmetry but relaxes the transitivity assumption. In particular, we show that transitivity can be weakened to consistency. This axiom is necessary and sufficient for the existence of a transitive and complete extension, even without requiring that any continuity properties be preserved by the extension. See Suzumura $(1976,1983)$ for this observation. Consistency requires that any preference cycle must be a cycle involving indifference only. 
Consistency: For all $x, y \in X$, for all $K \in \mathbb{N}$ and for all $x_{0}, \ldots, x_{K} \in X$, if $x=x_{0}$, $\left(x_{k-1}, x_{k}\right) \in R$ for all $k \in\{1, \ldots, K\}$ and $x_{K}=y$, then $(y, x) \notin P(R)$.

For an asymmetric relation, consistency is equivalent to $\mathrm{P}$-acyclicity, which requires that $P(R)$ does not exhibit cycles of any finite length. For relations that are not necessarily asymmetric, consistency implies acyclicity but the converse implication is not true.

We obtain

Theorem 2 Let $X$ be a topological space, and let $R \subseteq X \times X$ be a binary relation on $X$. If $R$ is asymmetric, consistent and upper semicontinuous, then $R$ has an upper semicontinuous strict order extension $R^{*} \subseteq X \times X$.

Proof The proof proceeds by showing that, given the properties of $R$ assumed in the theorem statement, the transitive closure $\bar{R}$ of $R$ is asymmetric and upper semicontinuous. Theorem 1 then allows us to conclude that $\bar{R}$ has an upper semicontinuous strict order extension $R^{*}$ and, because $R \subseteq \bar{R}, R^{*}$ is an upper semicontinuous strict order extension of $R$ as well. Therefore, the proof is complete after establishing those two properties of $\bar{R}$.

To prove that $\bar{R}$ is asymmetric, suppose, by way of contradiction, that there exist $x, y \in X, K, L \in \mathbb{N}$ and $x_{0}, \ldots, x_{K}, z_{0}, \ldots, z_{L} \in X$ such that $x=x_{0},\left(x_{k-1}, x_{k}\right) \in R$ for all $k \in\{1, \ldots, K\}, x_{K}=y, y=z_{0},\left(z_{\ell-1}, z_{\ell}\right) \in R$ for all $\ell \in\{1, \ldots, L\}$ and $z_{L}=x$. Because $R$ is asymmetric, we must have $\left(x_{k-1}, x_{k}\right) \in P(R)$ for all $k \in\{1, \ldots, K\}$ and $\left(z_{\ell-1}, z_{\ell}\right) \in P(R)$ for all $\ell \in\{1, \ldots, L\}$. Letting $M=K+L-1, w_{m}=x_{m}$ for all $m \in\{0, \ldots, K\}, w_{m}=z_{m-K}$ for all $m \in\{K+1, \ldots, K+L-1\}$ and $w=w_{K+L-1}$, we obtain $x=w_{0},\left(w_{m-1}, w_{m}\right) \in P(R)$ for all $m \in\{1, \ldots, M\}, w_{M}=x$ and $(w, x) \in P(R)$, contradicting the consistency of $R$.

To show that $\bar{R}$ is upper semicontinuous, let $x \in X$ be arbitrary, and let $y \in X$ be such that $(x, y) \in P(\bar{R})$. By definition of $\bar{R}$ and by the asymmetry of $R$, there exist $K \in \mathbb{N}$ and $x_{0}, \ldots, x_{K} \in X$ such that $x=x_{0},\left(x_{k-1}, x_{k}\right) \in P(R)$ for all $k \in\{1, \ldots, K\}$ and $x_{K}=y$. Because $R$ is upper semicontinuous, the set $\left\{z \in X \mid\left(x_{K-1}, z\right) \in P(R)\right\}$ is open. Furthermore, $x_{K}=y \in\left\{z \in X \mid\left(x_{K-1}, z\right) \in P(R)\right\}$. Therefore, there exists a neighborhood $\mathcal{N}(y)$ of $y$ such that $y^{\prime} \in\left\{z \in X \mid\left(x_{K-1}, z\right) \in P(R)\right\}$ for all $y^{\prime} \in \mathcal{N}(y)$. Thus, $\left(x, y^{\prime}\right) \in P(\bar{R})$ for all $y^{\prime} \in \mathcal{N}(y)$, which establishes that $\{z \in X \mid(x, z) \in P(\bar{R})\}$ is open. Thus, $\bar{R}$ is upper semicontinuous.

Unlike consistency, upper semicontinuity of an asymmetric relation $R$ is not necessary for the existence of an upper semicontinuous strict order extension of $R$, as demonstrated in the following example. 
Example 1 Let $X$ be the open interval $] 0,4[$ and suppose the relation $R$ is defined by

$$
R=\{(x, y) \in X \times X \mid x>y\} \backslash\{(3, y) \mid y \in] 1,2[\} .
$$

Because $\{y \in X \mid(3, y) \in P(R)\}=] 0,1] \cup[2,3[, R$ is not upper semicontinuous. Nevertheless, the relation

$$
R^{*}=\{(x, y) \in X \times X \mid x>y\}
$$

is an upper semicontinuous strict order extension of $R$.

\section{Extending general relations}

As mentioned in Remark 4 of Jaffray (1975, p. 402), the conclusion of Theorem 1 remains true if the assumption that $R$ is asymmetric is dropped (reflexivity can be added in its place but this is not necessary). In this section, we generalize this result by considering weakenings of transitivity.

First, note that upper semicontinuity together with consistency is not sufficient for the existence of an upper semicontinuous weak order extension if $R$ is not assumed to be asymmetric. Though consistency implies the existence of a weak order extension, consistency and upper semicontinuity together are not sufficient to guarantee that an upper semicontinuous extension can be found. Consider the following example.

Example 2 Let $X=] 0,4[$ and suppose the relation $R$ is defined by

$$
R=\{(1, x) \mid x \in X \backslash\{3\}\} \cup\{(x, 1) \mid x \in] 3,4[\} \cup\{(2,3),(3,2)\} .
$$

Because $P(R)=\{(1, x) \mid x \in] 0,1[\cup] 1,3[\}, R$ clearly is consistent. Furthermore, we have $\{y \in X \mid(1, y) \in P(R)\}=] 0,1[\cup] 1,3[$ and $\{y \in X \mid(x, y) \in P(R)\}=\emptyset$ for all $x \in X \backslash\{1\}$, which immediately implies that $R$ is upper semicontinuous. Suppose $R^{*}$ is a weak order extension of $R$. By definition of an extension, $(1,2) \in P(R)$ and $(2,3) \in I(R)$ imply $(1,2) \in P\left(R^{*}\right)$ and $(2,3) \in I\left(R^{*}\right)$. Because $R^{*}$ is transitive, we must have $(1,3) \in P\left(R^{*}\right)$. Because $R^{*}$ is a weak order extension of $R$, it follows that ] $0,1[\cup] 1,3] \subseteq\left\{y \mid(1, y) \in P\left(R^{*}\right)\right\}$ and $x \notin\left\{y \mid(1, y) \in P\left(R^{*}\right)\right\}$ for all $\left.x \in\right] 3,4[$. Hence, we obtain $\left.\left.\left\{y \mid(1, y) \in P\left(R^{*}\right)\right\}=\right] 0,1[\cup] 1,3\right]$ which is not open in $X$. Therefore, $R^{*}$ cannot be upper semicontinuous.

In order to obtain a set of sufficient conditions for the existence of an upper semicontinuous weak order extension, we add the following axiom to consistency and upper semicontinuity. 
IP\&PI-comparability: (i) For all $x, y, z \in X$, if $(x, y) \in I(R)$ and $(y, z) \in P(R)$, then $(x, z) \notin N(R)$.

(ii) For all $x, y, z \in X$, if $(x, y) \in P(R)$ and $(y, z) \in I(R)$, then $(x, z) \notin N(R)$.

IP\&PI-comparability is weaker than the conjunction of two of Sen's (1969) weakened transitivity conditions requiring that, whenever $(x, y) \in R$ and $(y, z) \in R$ with at least one strict preference, we have $(x, z) \in P(R)$. Note that Sen's conditions have strict preferences in the consequent rather than merely comparability. In the presence of consistency, IP\&PI-comparability and the conjunction of Sen's axioms IP and PI are equivalent.

Now we can prove

Theorem 3 Let $X$ be a topological space, and let $R \subseteq X \times X$ be a binary relation on $X$. If $R$ is consistent, IP\&PI-comparable and upper semicontinuous, then $R$ has an upper semicontinuous weak order extension $R^{*} \subseteq X \times X$.

Proof Suppose $R$ is consistent, IP\&PI-comparable and upper semicontinuous. The proof proceeds as follows. After establishing a consequence of consistency and IP\&PIcomparability, we show that there exists an asymmetric, consistent and upper semicontinuous relation on the quotient set of $X$ with respect to the transitive closure $\overline{I(R) \cup D}$ of $I(R) \cup D$. Then we invoke Theorem 2 to establish the existence of an upper semicontinuous strict order extension of that relation. Finally, we use this relation to define an upper semicontinuous weak order extension of $R$.

Step 1 We begin by showing that consistency and IP\&PI-comparability imply the following condition.

IP\&PI-transitivity: (i) For all $x, y, z \in X$, if $(x, y) \in \overline{I(R) \cup D}$ and $(y, z) \in P(R)$, then $(x, z) \in P(R)$.

(ii) For all $x, y, z \in X$, if $(x, y) \in P(R)$ and $(y, z) \in \overline{I(R) \cup D}$, then $(x, z) \in P(R)$.

To prove part (i) of $\overline{\mathrm{I}} \mathrm{P} \& \mathrm{P} \overline{\mathrm{I}}$-transitivity, let $x, y, z \in X$ be such that $(x, y) \in \overline{I(R) \cup D}$ and $(y, z) \in P(R)$. This implies that there exist $K \in \mathbb{N}$ and $x_{0}, \ldots, x_{K} \in X$ such that $x=x_{0},\left(x_{k-1}, x_{k}\right) \in I(R) \cup D$ for all $k \in\{1, \ldots, K\}$ and $x_{K}=y$. Consistency implies $\left(z, x_{K-1}\right) \notin R$. IP\&PI-completeness implies $\left(x_{K-1}, z\right) \notin N(R)$. Therefore, we must have $\left(x_{K-1}, z\right) \in P(R)$. Repeated application of this argument yields $(x, z) \in P(R)$. The proof of part (ii) is analogous.

Step 2 Clearly, $\overline{I(R) \cup D}$ is an equivalence relation. Let $X / \overline{I(R) \cup D}$ be the quotient set of $X$ with respect to $\overline{I(R) \cup D}$ and, for $x \in X$, let $\mathcal{I}_{x}=\{y \in X \mid(x, y) \in \overline{I(R) \cup D}\}$. We 
endow $X / \overline{I(R) \cup D}$ with the identification topology and, thus, a set $\mathcal{A} \subseteq X / \overline{I(R) \cup D}$ is open if and only if the set $\left\{x \in X \mid \mathcal{I}_{x} \in \mathcal{A}\right\}$ is open.

Step 3 Define a relation $\succ$ on $X / \overline{I(R) \cup D}$ as follows. For all $A, B \in X / \overline{I(R) \cup D}$,

$$
(A, B) \in \succ \Leftrightarrow \exists a \in A, b \in B \text { such that }(a, b) \in P(R)
$$

Step 4 Next, we show that $\succ$ has the required properties.

Step 4.a We begin by demonstrating that $\succ$ is an asymmetric relation, that is,

$$
P(\succ)=\succ
$$

That $P(\succ) \subseteq \succ$ is immediate. To prove the converse set inclusion, suppose that $A, B \in$ $X / \overline{I(R) \cup D}$ are such that $(A, B) \in \succ$. If $(A, B) \notin P(\succ)$, it follows that $(B, A) \in$ $\succ$. By definition, there exist $a, \bar{a} \in A$ and $b, \bar{b} \in B$ such that $(a, \bar{b}) \in P(R)$ and $(b, \bar{a}) \in P(R)$. Since $A$ and $B$ are equivalence classes of $\overline{I(R) \cup D},(\bar{a}, a) \in \overline{I(R) \cup D}$ and $(\bar{b}, b) \in \overline{I(R) \cup D}$. Now $\overline{\mathrm{I}} \& \mathrm{P} \overline{\mathrm{I}}$-transitivity implies $(a, b) \in P(R)$ and $(b, a) \in P(R)$, a contradiction. This proves (1).

Step 4.b To show that $\succ$ is consistent, suppose, by way of contradiction, that there exist $A, B \in X / \overline{I(R) \cup D}, K \in \mathbb{N}$ and $A_{0}, \ldots, A_{K} \in X / \overline{I(R) \cup D}$ such that $A=A_{0}$, $\left(A_{k-1}, A_{k}\right) \in \succ$ for all $k \in\{1, \ldots, K\}, A_{K}=B$ and $(B, A) \in P(\succ)$. There exist $a_{k}, \bar{a}_{k} \in A_{k}$ for all $k \in\{0, \ldots, K\}$ such that $\left(a_{k-1}, \bar{a}_{k}\right) \in P(R)$ for all $k \in\{1, \ldots, K\}$ and $\left(a_{K}, \bar{a}_{0}\right) \in P(R)$. Consider any $k \in\{1, \ldots, K\}$. If $\bar{a}_{k}=a_{k}$, we have $\left(a_{k-1}, a_{k}\right) \in P(R)$ by definition. If $\bar{a}_{k} \neq a_{k}$, it follows that $\left(\bar{a}_{k}, a_{k}\right) \in \overline{I(R) \cup D}$ because $\bar{a}_{k}$ and $a_{k}$ are in the same equivalence class $A_{k}$ according to $\overline{I(R) \cup D}$, and $\overline{\mathrm{I}} \& \mathrm{P} \overline{\mathrm{I}}$-transitivity implies $\left(a_{k-1}, a_{k}\right) \in P(R)$. Therefore, we obtain $\left(a_{k-1}, a_{k}\right) \in P(R)$ in all cases. Analogously, it follows that we must have $\left(a_{K}, a_{0}\right) \in P(R)$. But this contradicts the consistency of $R$.

Step 4.c To prove that $\succ$ is upper semicontinuous, we have to show that the set

$$
\{B \in X / \overline{I(R) \cup D} \mid(A, B) \in P(\succ)\}
$$

which, by (1), is equal to $\{B \in X / \overline{I(R) \cup D} \mid(A, B) \in \succ\}$, is open for all $A \in$ $X / \overline{I(R) \cup D}$. Let $A \in X / \overline{I(R) \cup D}$. By definition, $\{B \in X / \overline{I(R) \cup D} \mid(A, B) \in \succ\}$ is open if and only if

$$
\left\{x \in X \mid \mathcal{I}_{x} \in\{B \in X / \overline{I(R) \cup D} \mid(A, B) \in \succ\}\right\}
$$

is open. The set in (2) is equal to $\left\{x \in X \mid\left(A, \mathcal{I}_{x}\right) \in \succ\right\}$ which, in turn, is equal to $\left\{x \in X \mid \exists a \in A, b \in \mathcal{I}_{x}\right.$ such that $\left.(a, b) \in P(R)\right\}$. Let $a^{0} \in A$ be arbitrary. Because $A$ 
is an equivalence class according to $\overline{I(R) \cup D},\left(a^{0}, a\right) \in \overline{I(R) \cup D}$ and $\left(a, a^{0}\right) \in \overline{I(R) \cup D}$ for all $a \in A$. By part (i) of $\overline{\mathrm{IP}} \& \mathrm{P} \overline{\mathrm{I}}$-transitivity, we have

$$
(a, b) \in P(R) \Leftrightarrow\left(a^{0}, b\right) \in P(R)
$$

for all $a \in A$ and for all $b \in \mathcal{I}_{x}$. Analogously, because $\mathcal{I}_{x}$ is an equivalence class according to $\overline{I(R) \cup D},(b, x) \in \overline{I(R) \cup D}$ and $(x, b) \in \overline{I(R) \cup D}$ for all $b \in \mathcal{I}_{x}$. Now part (ii) of $\overline{\mathrm{IP}} \& \mathrm{P} \overline{\mathrm{I}}$-transitivity implies

$$
\left(a^{0}, b\right) \in P(R) \Leftrightarrow\left(a^{0}, x\right) \in P(R)
$$

for all $b \in \mathcal{I}_{x}$. (3) and (4) together imply

$$
\left\{x \in X \mid \exists a \in A, b \in \mathcal{I}_{x} \text { such that }(a, b) \in P(R)\right\}=\left\{x \in X \mid\left(a^{0}, x\right) \in P(R)\right\} .
$$

Because $R$ is upper semicontinuous, the latter set is open and, consequently, $\succ$ is upper semicontinuous as well.

Step 5 By Theorem 2, there exists an upper semicontinuous strict order extension $\succ^{*}$ of $\succ$. Let $\succ^{0}=\succ^{*} \cup\{(A, A) \mid A \in X / \overline{I(R) \cup D}\}$. Clearly, $\succ^{0}$ is a reflexive, transitive, complete and upper semicontinuous relation on $X / \overline{I(R) \cup D}$. Now define the relation $R^{*}$ on $X$ as follows. For all $x, y \in X$,

$$
(x, y) \in R^{*} \Leftrightarrow\left(\mathcal{I}_{x}, \mathcal{I}_{y}\right) \in \succ^{0}
$$

Step 6 It remains to be shown that $R^{*}$ has the desired properties.

Step 6.a $R^{*}$ is reflexive by definition, and $R$ is transitive and complete because $\succ^{0}$ is transitive and complete.

Step 6.b To prove that $R^{*}$ is a weak order extension of $R$, it is sufficient to show that $I(R) \subseteq I\left(R^{*}\right)$ and $P(R) \subseteq P\left(R^{*}\right)$. Let $(x, y) \in I(R)$. It follows that $(x, y) \in \overline{I(R) \cup D}$ and, thus, $\mathcal{I}_{x}=\mathcal{I}_{y}$. Because $\succ^{0}$ is reflexive, it follows that $\left(\mathcal{I}_{x}, \mathcal{I}_{y}\right) \in I\left(\succ^{0}\right)$ and, thus, $(x, y) \in I\left(R^{*}\right)$. If $(x, y) \in P(R)$, we obtain $\left(\mathcal{I}_{x}, \mathcal{I}_{y}\right) \in P\left(\succ^{0}\right)$ and, by definition, $(x, y) \in$ $P\left(R^{*}\right)$.

Step 6.c Finally, we prove that $R^{*}$ is upper semicontinuous. Let $x \in X$. We have

$$
\begin{aligned}
\left\{y \in X \mid(x, y) \in P\left(R^{*}\right)\right\} & =\left\{y \in X \mid\left(\mathcal{I}_{x}, \mathcal{I}_{y}\right) \in P\left(\succ^{0}\right)\right\} \\
& =\left\{y \in X \mid \mathcal{I}_{y} \in\left\{B \in X / \overline{I(R) \cup D} \mid\left(\mathcal{I}_{x}, B\right) \in P\left(\succ^{0}\right)\right\}\right\}
\end{aligned}
$$


which is open if and only if $\left\{B \in X / \overline{I(R) \cup D} \mid\left(\mathcal{I}_{x}, B\right) \in P\left(\succ^{0}\right)\right\}$ is open. Because $\succ^{0}$ is upper semicontinuous, the latter set is open and, consequently, $R^{*}$ is upper semicontinuous.

The axioms used in Theorem 3 are independent, as demonstrated in the following examples.

Example 3 Let $X=] 0,4[$ and

$$
\begin{aligned}
R & =\{(x, y) \mid x \in] 0,1[\text { and } y \in] 1,2[\} \\
& \cup\{(x, y) \mid x \in] 1,2[\text { and } y \in] 2,3[\} \\
& \cup\{(x, y) \mid x \in] 2,3[\text { and } y \in] 0,1[\} .
\end{aligned}
$$

$R$ satisfies IPEPI-comparability and upper semicontinuity but violates consistency.

The relation in Example 2 satisfies consistency and upper semicontinuity but violates IP\&PI-comparability.

Example 4 Let $X=] 0,2[$ and

$$
\begin{aligned}
R & =\{(x, y) \mid x \in] 0,1[\text { and } y \in] 1,2[\} \\
& \cup\{(x, y) \mid x, y \in] 0,1[\text { and } x \geq y\} \\
& \cup\{(x, y) \mid x, y \in[1,2[\text { and } x \geq y\}
\end{aligned}
$$

$R$ satisfies consistency and IPEPI-comparability but violates upper semicontinuity.

An alternative way of obtaining a set of sufficient conditions for the existence of an upper semicontinuous weak order extension is to add another continuity axiom to consistency and upper semicontinuity. This continuity property is defined as follows.

IP\&PI-continuity: (i) For all $x \in X$, the set $\{y \in X \mid \exists z \in X$ such that $(x, z) \in$ $I(R)$ and $(z, y) \in P(R)\}$ is open in $X$.

(ii) For all $x \in X$, the set $\{y \in X \mid \exists z \in X$ such that $(x, z) \in P(R)$ and $(z, y) \in I(R)\}$ is open in $X$.

IP\&PI-continuity does not imply and is not implied by upper semicontinuity (see the remark following the proof of the next theorem). However, if $R$ is reflexive (which we do not need to assume here), IP\&PI-continuity implies upper semicontinuity, as is easy to verify.

We obtain 
Theorem 4 Let $X$ be a topological space, and let $R \subseteq X \times X$ be a binary relation on $X$. If $R$ is consistent, upper semicontinuous and IPEPI-continuous, then $R$ has an upper semicontinuous weak order extension $R^{*} \subseteq X \times X$.

Proof Suppose $R$ is consistent, upper semicontinuous and IP\&PI-continuous. We proceed by constructing an extension $\hat{R}$ of $R$ that satisfies consistency, IP\&PI-comparability and upper semicontinuity, which allows us to invoke Theorem 3 to conclude that $\hat{R}$ (and, thus, $R$ ) has an upper semicontinuous weak order extension.

Step 1 Let $R_{0}=R$ and, for all $t \in \mathbb{N}$, define the relation $R_{t}$ recursively by

$$
\begin{array}{r}
R_{t}=R_{t-1} \cup\left\{(x, y) \mid \exists z \in X \text { such that }(x, z) \in I\left(R_{t-1}\right) \text { and }(z, y) \in P\left(R_{t-1}\right)\right. \text { or } \\
\left.(x, z) \in P\left(R_{t-1}\right) \text { and }(z, y) \in I\left(R_{t-1}\right)\right\} \text {. }
\end{array}
$$

Clearly,

$$
R_{t-1} \subseteq R_{t} \text { for all } t \in \mathbb{N}
$$

Now let $\hat{R}=\cup_{t \in \mathbb{N}_{0}} R_{t}$.

Step 2 We first prove that

$$
R_{t} \text { is consistent for all } t \in \mathbb{N}_{0} \text {. }
$$

We proceed by induction. By assumption, $R_{0}=R$ is consistent. Now suppose $R_{t-1}$ is consistent for $t \in \mathbb{N}$. By way of contradiction, suppose $R_{t}$ is not consistent. Then there exist $x, y \in X, K \in \mathbb{N}$ and $x_{0}, \ldots, x_{K} \in X$ such that $x=x_{0},\left(x_{k-1}, x_{k}\right) \in R_{t}$ for all $k \in\{1, \ldots, K\}, x_{K}=y$ and $(y, x) \in P\left(R_{t}\right)$. By definition of $R_{t}$, we have, for all $k \in\{1, \ldots, K\}$,

$$
\left(x_{k-1}, x_{k}\right) \in R_{t-1}
$$

or

$$
\exists y_{k} \in X \text { such that }\left(x_{k-1}, y_{k}\right) \in I\left(R_{t-1}\right) \text { and }\left(y_{k}, x_{k}\right) \in P\left(R_{t-1}\right)
$$

or

$$
\exists y_{k} \in X \text { such that }\left(x_{k-1}, y_{k}\right) \in P\left(R_{t-1}\right) \text { and }\left(y_{k}, x_{k}\right) \in I\left(R_{t-1}\right) \text {. }
$$

Furthermore, $\left(x_{K}, x_{0}\right) \in R_{t}$ implies

$$
\left(x_{K}, x_{0}\right) \in R_{t-1}
$$

or

$$
\exists y_{0} \in X \text { such that }\left(x_{K}, y_{0}\right) \in I\left(R_{t-1}\right) \text { and }\left(y_{0}, x_{0}\right) \in P\left(R_{t-1}\right)
$$


or

$$
\exists y_{0} \in X \text { such that }\left(x_{K}, y_{0}\right) \in P\left(R_{t-1}\right) \text { and }\left(y_{0}, x_{0}\right) \in I\left(R_{t-1}\right) .
$$

Because $\left(x_{0}, x_{K}\right) \notin R_{t}$, we obtain

$$
\left(x_{0}, x_{K}\right) \notin R_{t-1}
$$

and

$$
\nexists w_{0} \in X \text { such that }\left(x_{0}, w_{0}\right) \in I\left(R_{t-1}\right) \text { and }\left(w_{0}, x_{K}\right) \in P\left(R_{t-1}\right)
$$

and

$$
\nexists w_{0} \in X \text { such that }\left(x_{0}, w_{0}\right) \in P\left(R_{t-1}\right) \text { and }\left(w_{0}, x_{K}\right) \in I\left(R_{t-1}\right) .
$$

It follows that $\left(x_{K}, x_{0}\right) \in P\left(R_{t-1}\right)$ and, in all cases, we obtain a contradiction to the consistency of $R_{t-1}$.

Step 3 Next, we show that

$$
P\left(R_{t-1}\right) \subseteq P\left(R_{t}\right) \text { for all } t \in \mathbb{N}
$$

Suppose $(x, y) \in P\left(R_{t-1}\right)$ for some $t \in \mathbb{N}$. By (5), this implies $(x, y) \in R_{t}$. Suppose $(y, x) \in R_{t}$. By definition, this implies

$$
(y, x) \in R_{t-1}
$$

or

$$
\exists z \in X \text { such that }(y, z) \in I\left(R_{t-1}\right) \text { and }(z, x) \in P\left(R_{t-1}\right)
$$

or

$$
\exists z \in X \text { such that }(y, z) \in P\left(R_{t-1}\right) \text { and }(z, x) \in I\left(R_{t-1}\right) .
$$

The first possibility contradicts our hypothesis and the remaining two contradict the consistency of $R_{t-1}$. Therefore, $(x, y) \in P\left(R_{t}\right)$.

Step 4 Our next step is to prove

$$
I(\hat{R})=I(R)
$$

First, we prove by induction that

$$
I\left(R_{t}\right)=I(R) \text { for all } t \in \mathbb{N}_{0}
$$

By definition, $I\left(R_{0}\right)=I(R)$. Suppose $I\left(R_{t-1}\right)=I(R)$ for some $t \in \mathbb{N}$. Clearly, $I\left(R_{t-1}\right) \subseteq$ $I\left(R_{t}\right)$. Now suppose $(x, y) \in I\left(R_{t}\right)$. By definition, $(x, y) \in R_{t}$ implies

$$
(x, y) \in R_{t-1}
$$


or

$$
\exists z \in X \text { such that }(x, z) \in I\left(R_{t-1}\right) \text { and }(z, y) \in P\left(R_{t-1}\right)
$$

or

$$
\exists z \in X \text { such that }(x, z) \in P\left(R_{t-1}\right) \text { and }(z, y) \in I\left(R_{t-1}\right) \text {. }
$$

Because $(y, x) \in R_{t}$ as well, we obtain

$$
(y, x) \in R_{t-1}
$$

or

$$
\exists w \in X \operatorname{such} \text { that }(y, w) \in I\left(R_{t-1}\right) \text { and }(w, x) \in P\left(R_{t-1}\right)
$$

or

$$
\exists w \in X \text { such that }(y, w) \in P\left(R_{t-1}\right) \text { and }(w, x) \in I\left(R_{t-1}\right) .
$$

This implies $(x, y) \in I\left(R_{t-1}\right)$ because all other possibilities contradict (6). By the induction hypothesis, it follows that $I\left(R_{t}\right)=I(R)$. To complete the proof of (8), suppose $(x, y) \in I(\hat{R})$. By definition, there exist $t_{x}, t_{y} \in \mathbb{N}_{0}$ such that $(x, y) \in R_{t_{x}}$ and $(y, x) \in R_{t_{y}}$. Let $t=\max \left\{t_{x}, t_{y}\right\}$. By (5), it follows that $(x, y) \in R_{t}$ and $(y, x) \in R_{t}$ and, thus, $(x, y) \in I\left(R_{t}\right)=I(R)$.

Step 5 As a last preliminary result, we show that

$$
P(\hat{R})=\cup_{t \in \mathbb{N}} P\left(R_{t}\right)
$$

Suppose first that $(x, y) \in P(\hat{R})$. By definition, there exists $t^{\prime} \in \mathbb{N}$ such that $(x, y) \in R_{t^{\prime}}$ and there exists no $t^{\prime \prime} \in \mathbb{N}$ such that $(y, x) \in R_{t^{\prime \prime}}$. Therefore, we obtain $(y, x) \notin R_{t^{\prime}}$ and, thus, $(x, y) \in P\left(R_{t^{\prime}}\right) \subseteq \cup_{t \in \mathbb{N}} P\left(R_{t}\right)$.

Now suppose $(x, y) \in \cup_{t \in \mathbb{N}} P\left(R_{t}\right)$. Therefore, there exists $t^{\prime} \in \mathbb{N}$ such that $(x, y) \in R_{t^{\prime}}$ and $(y, x) \notin R_{t^{\prime}}$. By $(5),(y, x) \notin R_{t}$ for all $t<t^{\prime}$, and by $(7),(y, x) \notin R_{t}$ for all $t>t^{\prime}$. Therefore, $(y, x) \notin \hat{R}$ and, thus, $(x, y) \in P(\hat{R})$.

Step 6 We now show that $\hat{R}$ has the desired properties.

Step 6.a In light of (8), to prove that $\hat{R}$ is an extension of $R$, it remains to be shown that $P(R) \subseteq P(\hat{R})$. Suppose $(x, y) \in P(R)$. Because $R_{0}=R,(7)$ implies that $(x, y) \in P\left(R_{t}\right)$ for all $t \in \mathbb{N}_{0}$. Therefore, $(y, x) \notin \hat{R}$, and we obtain $(x, y) \in P(\hat{R})$.

Step 6.b Next, we prove that $\hat{R}$ satisfies consistency. Suppose not. Then there exist $x, y \in X, K \in \mathbb{N}$ and $x_{0}, \ldots, x_{K} \in X$ such that $x=x_{0},\left(x_{k-1}, x_{k}\right) \in \hat{R}, x_{K}=y$ and $(y, x) \in P(\hat{R})$. By definition of $\hat{R}$, there exist $t_{1}, \ldots, t_{K} \in \mathbb{N}_{0}$ such that $\left(x_{k-1}, x_{k}\right) \in R_{t_{k}}$ 
for all $k \in\{1, \ldots, K\}$. By (10), there exists $t_{0} \in \mathbb{N}_{0}$ such that $\left(x_{K}, x_{0}\right) \in P\left(R_{t_{0}}\right)$. Let $t=\max \left\{t_{0}, \ldots, t_{K}\right\}$. By (5) and (10), it follows that $\left(x_{k-1}, x_{k}\right) \in R_{t}$ for all $k \in\{1, \ldots, K\}$ and $\left(x_{K}, x_{0}\right) \in P\left(R_{t}\right)$, contradicting (6).

Step 6.c Now we show that $\hat{R}$ is IP\&PI-comparable. To establish part (i) of the property, suppose that $(x, y) \in I(\hat{R})$ and $(y, z) \in P(\hat{R})$ for some $x, y, z \in X$. By (8), it follows that $(x, y) \in I(R)$. By (10), there exists $t \in \mathbb{N}_{0}$ such that $(y, z) \in P\left(R_{t}\right)$. By (9), $(x, y) \in I\left(R_{t}\right)$. Therefore, $(x, z) \in R_{t+1}$ by definition and, thus, $(x, z) \in \hat{R}$ which implies $(x, z) \notin N(\hat{R})$. The proof of part (ii) is analogous.

Step 6.d To show that $\hat{R}$ is upper semicontinuous, we begin by establishing that $R_{t}$ satisfies upper semicontinuity and IP\&PI-continuity for all $t \in \mathbb{N}_{0}$. Again, we proceed by induction. By assumption, $R=R_{0}$ is upper semicontinuous and IP\&PI-continuous. Now suppose $R_{t-1}$ has those two properties for some $t \in \mathbb{N}$, and let $x, y \in X$ be such that $(x, y) \in P\left(R_{t}\right)$. By definition, it follows that

$$
(x, y) \in R_{t-1}
$$

or

$$
\exists z \in X \text { such that }(x, z) \in I\left(R_{t-1}\right) \text { and }(z, y) \in P\left(R_{t-1}\right)
$$

or

$$
\exists z \in X \text { such that }(x, z) \in P\left(R_{t-1}\right) \text { and }(z, y) \in I\left(R_{t-1}\right) .
$$

In the first case, the upper semicontinuity of $R_{t-1}$ implies that there exists a neighborhood $\mathcal{N}(y)$ of $y$ such that $y^{\prime} \in\left\{w \in X \mid(x, w) \in P\left(R_{t-1}\right)\right\}$ for all $y^{\prime} \in \mathcal{N}(y)$. By (7), it follows that $y^{\prime} \in\left\{w \in X \mid(x, w) \in P\left(R_{t}\right)\right\}$ for all $y^{\prime} \in \mathcal{N}(y)$. In the second and third cases, the same conclusion is reached by invoking the IP\&PI-continuity rather than the upper semicontinuity of $R_{t-1}$. Thus, the set $\left\{w \in X \mid(x, w) \in P\left(R_{t}\right)\right\}$ is open in $X$ for all $x \in X$ and, therefore, $R_{t}$ is upper semicontinuous. To prove that $R_{t}$ satisfies part (i) of IP\&PI-continuity, suppose $(x, z) \in I\left(R_{t}\right)$ and $(z, y) \in P\left(R_{t}\right)$ for some $x, y, z \in X$. Because $R_{t}$ is upper semicontinuous, there exists a neighborhood $\mathcal{N}(y)$ of $y$ such that $y^{\prime} \in\left\{w \in X \mid(z, w) \in P\left(R_{t}\right)\right\}$ for all $y^{\prime} \in \mathcal{N}(y)$. Hence, the set $\{y \in X \mid \exists z \in$ $X$ such that $(x, z) \in I(R)$ and $(z, y) \in P(R)\}$ is open in $X$ for all $x \in X$. The proof that $R_{t}$ satisfies part (ii) of IP\&PI-continuity is analogous.

Let $x \in X$. By (10),

$$
\begin{aligned}
\{y \in X \mid(x, y) \in P(\hat{R})\} & =\left\{y \in X \mid y \in \cup_{t \in \mathbb{N}}\left\{z \in X \mid(x, z) \in P\left(R_{t}\right)\right\}\right\} \\
& =\cup_{t \in \mathbb{N}}\left\{y \in X \mid(x, y) \in P\left(R_{t}\right)\right\} .
\end{aligned}
$$


As shown above, $\left\{z \in X \mid(x, z) \in P\left(R_{t}\right)\right\}$ is open in $X$ for all $x \in X$ and for all $t \in \mathbb{N}_{0}$ and, thus, $\{y \in X \mid(x, y) \in P(\hat{R})\}$ is the union of a collection of open sets, which is itself open. Therefore, $\hat{R}$ is upper semicontinuous.

Step 7 By Theorem 3, $\hat{R}$ has an upper semicontinuous weak order extension $R^{*}$ and, because $\hat{R}$ is an extension of $R, R^{*}$ is an upper semicontinuous weak order extension of $R$ as well.

The same examples as those following Theorem 3 establish that the axioms used in Theorem 4 are independent.

As mentioned earlier, consistency is not only sufficient but also necessary for the existence of a transitive and complete extension. However, none of the other axioms used in Theorems 3 and 4 are necessary for the existence of an upper semicontinuous weak order extension. Consider the following example.

Example 5 Let $X=] 0,4[$ and suppose the relation $R$ is defined by

$$
R=\{(x, y) \in X \times X \mid x \geq y \text { or } x, y \in[1,2]\} \backslash\{(3, y) \mid y \in] 0,1[\cup\{2\}\} .
$$

Because the set $\{y \in X \mid(3, y) \in P(R)\}=[1,2[\cup] 2,3[$ is not open, $R$ is not upper semicontinuous. Furthermore, because $(3,1) \in P(R),(1,2) \in I(R)$ and $(3,2) \in N(R)$, $R$ is not IPESP-comparable. Because $R$ is reflexive, the fact that upper semicontinuity is violated implies that $R$ is not IPEPI-continuous (see the remark following the definition of IPEPI-continuity). However, the relation

$$
R^{*}=\{(x, y) \in X \times X \mid x \geq y \text { or } x, y \in[1,2]\}
$$

is an upper semicontinuous weak order extension of $R$.

\section{Concluding remarks}

In concluding this paper, two remarks are in order. In the first place, noncomparabilities as discussed in the introduction may be eliminated as the decision maker accumulates experience, but this process is usually path-dependent: any weak order extension may be the one finally reached. Thus, an interesting application of our results consists of combining them with those of Suzumura and $\mathrm{Xu}$ (2001). In particular, we examine the relationship between choices made on the basis of an underlying incomplete and intransitive preference relation and choices that result from various weak order extensions of this relation. 
Suppose $R$ is a binary relation defined on a topological space $X$. Let $\mathcal{B}$ be the set of nonempty and compact subsets of $X$. For all $B \in \mathcal{B}$, the set of $R$-maximal elements in $B$ is $M(R, B)=\{x \in B \mid(y, x) \notin P(R)$ for all $y \in B\}$, and the set of $R$-greatest elements in $B$ is $G(R, B)=\{x \in B \mid(x, y) \in R$ for all $y \in B\}$. Let $\Omega(R)$ be the set of all upper semicontinuous weak order extensions of $R$. According to Suzumura and $\mathrm{Xu}$ (2001), a relation $R$ is choice-functionally recoverable if $M(R, B)=\cup_{R^{*} \in \Omega(R)} G\left(R^{*}, B\right)$ for all $B \in \mathcal{B}$.

Suppose $R$ is consistent, upper semicontinuous and IP\&PI-comparable (resp. IP\&PIcontinuous). This implies that the set of $R$-maximal elements in $B$ is nonempty for all $B \in \mathcal{B}$. Applying our Theorem 3 (resp. Theorem 4 ), it follows that $\Omega(R)$ is nonempty. Now Theorem 3.2 of Suzumura and $\mathrm{Xu}$ (2001) implies that $R$ is choice-functionally recoverable if and only if $\cap_{R^{*} \in \Omega(R)} P\left(R^{*}\right) \subseteq R$.

In the second place, we focus in this paper on topological spaces in order to examine the possibilities of extending incomplete relations in a way that preserves upper semicontinuity. With some additional structure imposed on the universal set under consideration, further interesting questions could be addressed. For example, in addition to (or instead of) upper semicontinuity, one might want to require an extension to possess classical economic properties of preferences such as monotonicity or convexity. The exploration of these further issues is left for future research.

\section{References}

Aumann, R. (1962), "Utility theory without the completeness axiom," Econometrica 30, 445-462.

Bossert, W. (1999), "Intersection quasi-orderings: an alternative proof," Order 16, 221-225.

Donaldson, D. And J.A. Weymark (1998), "A quasiordering is the intersection of orderings," Journal of Economic Theory 78, 382-387.

DugGan, J. (1999), "A general extension theorem for binary relations," Journal of Economic Theory 86, 1-16.

Dushnik, B. AND E.W. Miller (1941), "Partially ordered sets," American Journal of Mathematics 63, 600-610.

FishbuRn, P.C. (1970), Utility Theory for Decision Making, Wiley, New York. 
Fishburn, P.C. (1973), The Theory of Social Choice, Princeton University Press, Princeton.

Hansson, B. (1968), "Choice structures and preference relations," Synthese 18, 443-458. JAFfray, J.-Y. (1975), "Semicontinuous extension of a partial order," Journal of Mathematical Economics 2, 395-406.

Sen, A.K. (1969), "Quasi-transitivity, rational choice and collective decisions," Review of Economic Studies 36, 381-393.

Suzumura, K. (1976), "Remarks on the theory of collective choice," Economica 43, 381-390.

Suzumura, K. (1983), Rational Choice, Collective Decisions and Social Welfare, Cambridge University Press, New York.

Suzumura, K. And Y. Xu (2001), "Recoverability of choice functions and binary relations: some duality results," mimeo, Institute of Economic Research, Hitotsubashi University.

SzPilrajn, E. (1930), "Sur l'extension de l'ordre partiel," Fundamenta Mathematicae 16, 386-389. 